\title{
Rasgos de simplificación en el habla rural de dos localidades de Chile: descripción fonotáctica y discursiva*
}

\author{
Doctora Miriam Elizabeth Cid Uribe** \\ Doctoranda Macarena Céspedes Morales***
}

\section{Resumen:}

El habla rural de Chile, variedad dialectal recurrente en alrededor del $14 \%$ de la población del país, según el último censo nacional, manifiesta rasgos particulares a nivel fonotáctico y discursivo que la caracterizan y la diferencian de otras variedades dialectales recurrentes en el país. Dentro de los aspectos fonotácticos se encuentran simplificaciones segmentales -elisiones, asimilaciones, sinalefas, sinéresis, sustituciones, entre otros- a las que se recurre con mayor o menor frecuencia; en el aspecto discursivo, la presencia y recurrencia de pausas llenas o vacías, las partidas falsas, las repeticiones, el alargamiento segmental se manifiestan en forma constante en esta variedad. En este artículo damos cuenta del comportamiento de los rasgos de simplificación en el habla rural de dos localidades de la Región Metropolitana desde el punto de vista de la manifestación fonotáctica de cada uno de estos rasgos en la elaboración de su discurso semiespontáneo.

Palabras Clave: Habla rural, fonotáctico, discursivo, simplificaciones, pausa, alargamiento

\section{Abstract:}

Chilean rural speech, variety spoken by nearly $14 \%$ of the population of the country according to the latest national census, shows specificphonotactic and discourse features which characterise and differentiate it from other dialectal varieties found in the country. Segmental simplifications like elision, assimilation, gradation, compression, substitution, amongst others, are to be found as recurrent features at the phonotactic level; at the discourse level, phenomena like pauses -filled or empty-, false starts, repetitions, segmental lengthening are recurrent in this variety. In this article we give an account of the behaviour of such features as they are manifested -phonotactically and discoursally-in two rural communities of the Metropolitan Region at the moment of producing semi spontaneous speech.

Key words: Rural speech, phonotactic, discourse, simplifications, pause, lengthening

* Parte de los resultados presentados en este artículo han sido tomados de la tesis de Magíster "Descripción fonofonológica de habla rural de dos localidades de un mismo sector de la zona central de Chile", realizada por Macarena Céspedes en el Programa de Magíster en Lingüística de la Facultad de Letras, Pontificia Universidad Católica de Chile.

** Actualmente académica de la Pontificia Universidad Católica de Chile. mcidu@uc.cl

*** Actualmente académica de la Universidad Católica Silva Henríquez. mlcesped@uc.cl 
Rasgos de simplificación en el habla rural de dos localidades de Chile: descripción fonotáctica y discursiva / Dras@ Miriam Cid Uribe y Macarena Céspedes Morales

\section{Introducción}

Para el hombre de la calle, para aquel que no ha tenido una instrucción formal ni informal específica acerca de los procesos fonológicos que caracterizan y diferencian las variedades dialectales del español hablado en Chile, no es ningún misterio que existen personas que hablan como "huasos", que hablan como "campesinos", que son, en otras palabras, usuarios de una variedad que los identifica como "rurales" o "campesinos". Para el erudito, por otra parte, para aquel que sí se ha preocupado de analizar las variedades dialectales presentes en nuestro país, la existencia de un habla Ilamada "rural", tampoco lo es. Obviamente, los caminos para llegar a este punto de confluencia son diferentes: para el primero, se trata de mera observación y registro auditivo; para el segundo, se trata de análisis científicos, medibles, cuantificables y descriptibles, de muestras que reflejan características propias de cada variedad. Así las cosas, podríamos tal vez hacer un pequeño ejercicio a través del cual lograremos -dentro de las limitantes propias del mismo- acercarnos a ciertos aspectos fundamentales que nos servirán como marco referencial. Dicho ejercicio consistirá en intentar una identificación precisa de aquellos elementos -segmentales, prosódicos, discursivos-que reflejen la calidad de "huasa" del habla campesina. De partida, nos encontraremos con dificultades por cuanto aquellos aspectos identificatorios parecen escapar de la verbalización para caer simplemente en la apreciación subjetiva: identificamos el habla rural no sólo por el léxico recurrente, por las estructuras gramaticales en que se inserta dicho léxico, por los alcances semánticos de ítemes léxicos utilizados sino por algo más: no es lo que dice, sino el cómo lo dice lo que identifica al hablante rural. Pareciera haber un conglomerado de elementos que, en conjunto, caracterizan esta variedad y dentro de estos elementos los rasgos de simplificación alcanzan, a nuestro juicio, gran preeminencia.

\subsection{Rasgos de simplificación}

Los rasgos de simplificación fonológica han sido observados tradicionalmente desde el punto de vista de los trastornos del habla (Jakobson, 1941) o desde el punto de vista puramente fonético (Canellada y Kuhlman Madsen, 1987) como rasgos que caracterizan ciertas manifestaciones del habla en entornos de discursos particulares. A nuestro juicio, los rasgos de simplificación, entendidos como fenómenos del habla continua y, por ende, propios de la oralidad y recurrentes en la misma, pueden y deben ser analizados desde una perspectiva fonofonológica; en ella, el comportamiento de tales rasgos en distintas situaciones comunicativas adquiere importancia a la hora de entregar una descripción de habla propia de la variedad motivo de este estudio: la variedad rural del español de Chile. Los rasgos que nos interesan tienen, a nuestro juicio, un comportamiento 
característico en esta variedad que la diferencia del habla estándar de Chile en grados y alcances que requieren de una explicitación detallada que consigne ocurrencias, recurrencias condicionantes combinatorias, por nombrar solo algunos aspectos.

Consideramos los rasgos de simplificación, como lo dijéramos en párrafos anteriores, no sólo como rasgos que caracterizan una variedad desde el punto de vista gramatical -elisiones de artículos o verbos, por ejemplo- o desde el punto de vista léxico -ausencia de sinónimos, con la consecuente evidencia de pobreza léxica, por nombrar solo uno-, sino también como recursos fonotácticos los que, siguiendo reglas fonofonológicas sistémicas propias de la variedad en estudio, hacen del habla producida un todo discursivo coherente, cohesionado y fluido identificable como propio y particular del habla rural de Chile. Dentro de las simplificaciones encontradas en el habla de informantes provenientes de dos localidades rurales de Chile -punto de partida para la investigación de la cual damos cuenta en este artículo-y adscribiendo a los postulados desarrollados en la introducción del mismo, dividiremos los rasgos que nos interesan en dos grandes grupos en consideración a su particular comportamiento lingüístico: los fonotácticos, directamente relacionados con la estructura interna del enunciado, y los discursivos que dicen relación con fenómenos que afectan la totalidad del texto oral.

\subsubsection{Elementos fonotácticos}

Adscritos al sistema segmental de la variedad bajo estudio, los elementos fonotácticos reflejan, por una parte, posibilidades combinatorias de los segmentos en determinados entornos fonofonológicos; por otra, las potencialidades de introducir modificaciones en la cadena hablada de forma tal que las estructuras silábicas -por nombrar un elemento significativo- se ven alteradas en su composición. En este grupo, alcanzan especial preponderancia los fenómenos de diptongación -el cual generalmente surge por sinalefa en grupos fónicos adyacentes y por sinéresis al interior del grupo-; de elisión, tanto de segmentos como de sílabas; de asimilación tanto progresiva como regresiva y recíproca; y de sustitución de segmentos, generalmente manifestada en sustitución de consonante por vocal y, en menor grado, sustitución de una vocal por otra.

\subsubsection{Elementos discursivos}

En este grupo, podemos distinguir aquellos rasgos a los que el hablante recurre con el fin de hacer de sus enunciados secuencias coherentes y cohesivas. Entre los más recurrentes se evidencian los alargamientos segmentales, las pausas llenas o vacías -estas últimas generalmente precedidas por un movimiento tonal ascendente indicador prosódico universal de la intención del hablante de mantener su turno discursivo-, 
Rasgos de simplificación en el habla rural de dos localidades de Chile: descripción fonotáctica y discursiva / Dras@ Miriam Cid Uribe y Macarena Céspedes Morales

las partidas falsas y las repeticiones. Especial importancia adquieren aquí los aspectos prosódicos, sobre todo la entonación y el ritmo, que acompañan a los enunciados y caracterizan el habla bajo estudio.

\subsection{Habla rural de Chile}

El concepto Habla Rural de Chile ha de ser entendido como una variedad dialectal del español hablado en este país que está presente en una determinada comunidad de habla, la rural, la cual surge por una cuestión geográfica e histórica. De acuerdo a la información recabada en el último censo nacional llevado a cabo en el año 2002, un $14 \%$ de la población de Chile es descriptible como rural. Sin embargo, el habla característica de este segmento de la población no ha sido objeto, últimamente, de atención lingüística en un grado importante. Así, la bibliografía revisada respecto al tema refleja claramente una carencia en aspectos importantes tales como (1) una descripción holística del concepto operacional de habla rural y (2) descripciones actualizadas del comportamiento fonofonológico de los hablantes rurales de nuestro país. De hecho, hemos podido constatar que los estudios dialectales referidos a la variedad que nos interesa han sido abordados desde perspectivas que van de lo léxico a lo gramatical y, sólo en años recientes, a lo fonofonológico.

\subsection{La investigación}

En este contexto general, y ante la necesidad de acceder a información que nos permita proponer una caracterización fonofonológica actualizada del habla rural de Chile, se realiza un estudio de habla de dos localidades de una misma región del país. Dicho estudio se sustenta en la hipótesis de que "el habla rural presente en las localidades de Culiprán y Popeta no tiene comportamientos fonofonológicos diferentes entre sí; no obstante, el habla producida en estas localidades presenta rasgos propios y características de tipo fonofonológicos que la identifican como diferente al habla estándar del país". En este artículo interesa realizar una primera aproximación a una descripción holística del habla rural de Chile, y postular, a la luz de los datos reflejados en tal descripción, una definición operacional del concepto que sustenta esta investigación.

\section{Antecedentes generales}

En el entorno Latinoamericano general, el habla rural ha sido un área de importancia para los estudios dialectales. Hemos constatado que en Venezuela, al igual que en otros países como México y Colombia, por ejemplo, las investigaciones sobre el tema tienden a enmarcarse en una perspectiva sociolingüística y tienden a hacer postulados acerca de la 
particular relación entre la lingüística y la sociolingüística adscribiendo importancia crucial a la última por cuanto el "lenguaje es un ente social" 1 . En Venezuela se han elaborado tres corpora, que consisten en materiales hablados de Caracas, Maracaibo y Mérida. Las grabaciones recopiladas en tales corpora han sido transcritas de manera ortográfica, son genéricas y posibilitan la investigación parcial según el interés particular de cada investigador interesado en el tema. Los estudios resultantes de los análisis de los corpora mencionados, se han traducido en descripciones circunscritas a factores sociales, a factores situacionales, a factores etáreos, a factores de género, por ejemplo. En este sentido, cabe mencionar la investigación Las variantes fonéticas del habla de la cordillera de Mérida: ¿Cambio lingüístico o patrón de sexo?, que analiza el comportamiento lingüístico de hombres y mujeres y postula como conclusión que las mujeres optan por los rasgos del habla urbana que es más prestigiosa (y prefieren irse a vivir allí) y que los hombres jóvenes no dejan de usar los rasgos rurales. Esta explicación la respalda el hecho de que hombres y mujeres tienen, en la cosmovisión de los habitantes de la zona, funciones muy distintas (Álvarez, 1998). Desde otra perspectiva, importa destacar el trabajo de Enrique Obediente, Fonetismo Segmental en el español hablado de Venezuela, quien postula que no todo el país constituye un área homogénea y que parte de su territorio -región andina, específicamente- presenta algunos rasgos de las tierras altas especialmente el consonantismo ${ }^{2}$. El artículo en general describe sintéticamente lo más relevante del fonetismo segmental de la norma culta urbana en el habla espontánea, pero también se detiene y muestra ciertas particularidades que se dan en otros registros de habla o en otros grupos sociales. Importa para este artículo destacar la postura de Obediente acerca del habla rural del español hablado en Venezuela cuando postula que dicha variedad dialectal presenta características únicas y excluyentes que la identifican como diferente del habla urbana.

\subsection{Comunidades Lingüísticas}

Nuestro campo de registro es el mundo rural, el campo, en donde existe otra marcación lingüística en el habla de quienes allí habitan; se observa el mismo código español que rige nuestra lengua castellana, pero se evidencias diferencias importantes en todos los niveles lingüísticos.

Es sabido que la lengua es variable y que se produce de manera variable. Ahora, esa variabilidad pareciera surgir de factores de tipo esencialmente lingüísticos, pero que aparecen acompañados por otros que son intrínsecamente sociológicos. Frente a esto, Moreno Fernández (2005), nos explica la variable lingüística como el elemento, rasgo o unidad

\footnotetext{
Álvarez, Alexandra. Departamento de Lingüística de la Universidad de Los Andes, Mérida.

2 Obediente, Enrique. Fonetismo Segmental. Universidad de Los Andes.
} 
Rasgos de simplificación en el habla rural de dos localidades de Chile: descripción fonotáctica y discursiva / Dras@ Miriam Cid Uribe y Macarena Céspedes Morales

lingüística que puede manifestarse de formas distintas; por lo tanto, de manera variable. Así mismo, cada una de las expresiones de una variable recibe el nombre de variante lingüística; se insiste en que la variación, definida como el uso alterno de formas diferentes de decir lo mismo, se puede encontrar prácticamente en todos los niveles de la lengua, desde la fonética y fonología hasta el discurso. Para nosotros es de suma importancia llegar a un claro entendimiento y comparación de variación, y cómo esto surge de variables lingüísticas y sociológicas dadas en una localidad (sociocultural) en donde no se concentra la lengua canónica, sino lo que conocemos como habla rural. Según López Morales, los factores lingüísticos que pueden determinar una variación pueden ser divididos en tres grupos: distribucionales (lugar en que aparece el fonema o morfema), contextuales (elementos antecesores o sucesores de la variable) y funcionales (inclusión de la variable en categorías gramaticales) (Moreno Fernández, 2005; 26). La variación fonética fonológica se ve determinada por factores lingüísticos y extralingüísticos, y es en estos últimos donde intervienen factores ajenos a la lengua y que son mayoritariamente factores sociales. En consecuencia, es necesario el estudio de la variable "clase social" compuesta por una serie de clasificaciones sociales y de sociedades. De los estudios sociolingüísticos que enfrentan esta variable está el de W. Labov, quien reflexiona al respecto; en muchas comunidades existe una clara conciencia de que existe 'algo' que clasifica y distingue a los individuos por estratos: los hablantes se sienten miembros de una clase, no siempre satisfechos, y se consideran capaces de clasificar socialmente a otros hablantes. Así, cada hablante urbano puede notar, por medio del lenguaje, cuando un poblador rural llega a la ciudad; así mismo un hablante centro urbano nota cuando el nuevo interlocutor es marginal, es decir, que es periférico. Esta discriminación sociolingüística tiene directa relación con el sociolecto que maneje el hablante, porque éste sabe reconocerse o no en el habla del otro. Se entiende por sociolecto al conjunto de características lingüísticas propias de un grupo, estrato o clase. El sociolecto está muy ligado a las variedades dialectales que sobrellevan variables sociales como educación, nivel o grado de instrucción y estudios o escolaridad.

\section{Análisis descriptivo de los rasgos fonotácticos y discursivos, presentes en el habla rural de Culiprán y Popeta}

\subsection{Culiprán y Popeta: localidades rurales}

La descripción que se presenta en este artículo se basa en resultados obtenidos del análisis del habla de hombres (8) y mujeres (8) de las loca- 
lidades rurales de Culiprán y Popeta, ambas pertenecientes a la Provincia de Melipilla, Región Metropolitana.

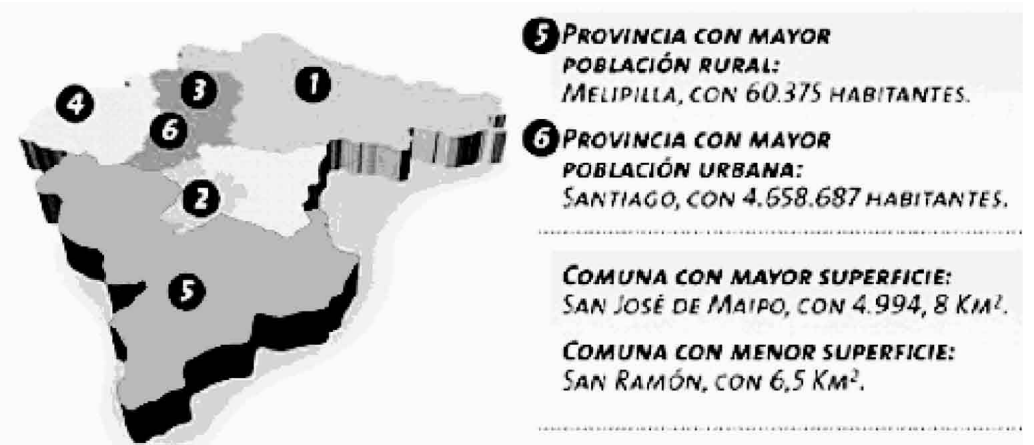

Fig. 1. Melipilla-Región Metropolitana (MOP / Síntesis regional 2007)

Culiprán es parte de la Provincia de Melipilla que está ubicada a 64 kilómetros al sur de Santiago; Culiprán se ubica a doce kilómetros al sur de Melipilla, capital de la provincia. Dicha capital limita en todos sus puntos con zonas rurales parecidas en su extensión y características geográficas generales. Los límites de la ciudad de Melipilla son: Al Norte y Noroeste, la localidad de Chocalán; al Noreste, Pabellón; al Este, Cholqui; y al Sureste, Tantehue. Culiprán, por su parte, limita al Norte con el Cordón de Cerros de Culiprán; al Sur con Popeta; al Suroeste con parte de Popeta y Mandinga; y al Oeste con San Manuel. Su superficie es de 7126,8 hectáreas según escritura del Registro de Propiedades del año 1966. ${ }^{3}$ Culiprán, como localidad rural mayor en términos de habitantes, está dividida en nueve sectores: al Norte, El Molino; al Noreste, La Alianza; al Este, Culiprán (conocido popularmente como La calle Central); al Sureste, El Progreso y Los Molles; al Sur, Las Chilcas y Villa Mi Casa, y al Noroeste, El Portezuelo. Desde el punto de vista demográfico, Culiprán cuenta con una población total de 1.986 habitantes, de los cuales 1.055 son hombres y 931 son mujeres (Censo Nacional, 2002). La actividad económica de los habitantes de Culiprán se concentra mayoritariamente en la agricultura y la ganadería; los cultivos predominantes son el choclo, la papa y los tomates, mientras que en la ganadería se privilegia la crianza de ganado vacuno. En segundo lugar, están las actividades económicas correspondientes a los servicios: labores de secretariado, empleos domésticos, y ocasionalmente algunos puestos de carácter profesional con alto grado académico. En último lugar se encuentra la actividad industrial, representada por agroindustrias relativamente cercanas a Culiprán. 
Popeta, por su parte y al igual que Culiprán, es otra localidad rural ubicada en la Región Metropolitana de Santiago, Provincia de Melipilla, comuna de Melipilla. Limita en todos sus puntos con zonas rurales parecidas en su extensión y características geográficas generales. Sus límites son: Al Norte, Culiprán; al Este, San Juan de Popeta; al Oeste, Mandinga y Codigua; y al Sur, Los Maitenes y Longovilo. Desde el punto de vista demográfico, Popeta cuenta con una población total de 917 habitantes, de los cuales 479 son hombres y 438 son mujeres (Censo Nacional, 2002). La actividad económica desarrollada en Popeta es semejante a la de Culiprán, con excepción a la actividad agroindustrial que alcanza mayor relevancia en Popeta.

\subsection{Rasgos fonotácticos en el habla rural de Culiprán y Popeta}

Los rasgos de simplificación, más recurrentes, encontrados en el corpus de habla transliterada, son la elisión, sinalefa, sinéresis y sustitución. A continuación daremos a conocer la descripción y función discursiva de cada uno de ellos:

A. La elisión segmental es conocida como el alófono cero que se presenta como una variante de un fonema determinado, cuya ocurrencia no se realiza. Dentro de la muestra se detectaron elisión de /s/, /d/ y /b/ en contexto intervocálico y al final de grupo tónico en los casos de /s/y/d/, por ejemplo:
1. /traba 'xaba/ $\rightarrow$ [traфa ' xaфa] 'trabajaba'
2. /xuben 'tud/ $\rightarrow$ [xußen 'tu $\phi]$ 'juventud'
3. /mut $\int o s / \rightarrow[$ 'mufoф] 'muchos'

Este rasgo se manifiesta, también y con una alta frecuencia, como la elisión de sílaba al inicio, interior o cierre de grupo fónico; este caso consiste en el acortamiento de las palabras, generando, así, una sinalefa dentro del grupo fónico.

B. Entendemos por sinalefa el fenómeno que une un grupo tónico con otro y que ocurre dentro del grupo fónico, además, tendremos en cuenta la diferencia que existe entre una sinalefa por elisión y una por diptongación. La sinalefa por elisión se da cada vez que existe un caso de hiato entre dos grupos tónicos, en estas circunstancias tienden a perderse las vocales abierta, semiabierta o semicerrada, resultando de ambos un único grupo tónico. Se observó la presencia de sinalefas producidas principalmente por la elisión de sílaba cerrada por la consonante s que tiende a ser aspirada, como se observa en los siguientes ejemplos: 
1. 'ahora están' $\rightarrow$ "ahora(e)htán"

2. 'lo estoy' $\rightarrow$ "lo(e)htoy"

3. 'ya estamos' $\rightarrow$ "ya(e)htamos"

4. 'como estar' $\rightarrow$ "como(e)htar"

En esta variedad se presencia, en forma distinta, ocurrencias de elisión que corresponden a una sinalefa que forma un sirrema (Quilis, 1993: 372) dentro de los enunciados, por ejemplo:

1. 'Están jugando' $\rightarrow$ "(es)tán jugando" $\rightarrow$ [tanxu 'vando]

2. 'Manos atadas' $\rightarrow$ "manos ata(da)s" $\rightarrow$ [manoфa 'tah]

3. 'Estoy bajoneada' $\rightarrow$ "(es)toy bajonea(da)" $\rightarrow$ [toivaxo ' nea]

4. 'Para que' $\rightarrow$ "pa(ra)que" $\rightarrow$ [pa ' ke]

5. 'Como estar' $\rightarrow$ "como (es)tar" $\rightarrow$ [komoh ' tar]

6. 'Está bien' $\rightarrow$ "(es)tá bien" $\rightarrow$ [ta ' $\beta$ jen]

7. 'Está mal' $\rightarrow$ "(es)ta mal" $\rightarrow$ [ta ' mal]

8. 'Parece que' $\rightarrow$ "pare $(c e) q u e^{\prime \prime} \rightarrow$ [pa 'reke]

Estos casos corresponden a elisiones silábicas que reducen una sílaba final o inicial de palabras quedando átonas en su totalidad, estas sílabas átonas se amalgaman con la palabra que sigue a continuación adquiriendo de esta manera un núcleo silábico diferente al esperado.

Concluimos que la sinalefa, en muchos de los casos estudiados, contribuye a la formación de palabras que proyectan verdaderos neologismos, como por ejemplo: 1. Para + el = "pal"

2. Para + arriba $=$ "parriba"

3. Para + abajo = "pabajo"

4. Para + atrás = "patrás"

A estos ejemplos, se suman otros que se forman debido a la elisión de sílaba al interior de palabra, como es el caso de la palabra "catolihmo" que surge de la elisión de la sílaba tónica cis existente en la palabra catolicismo, que fue encontrada en el corpus descrito.

Al hablar de sinalefa por diptongación, nos referimos al caso en que la unión de dos segmentos vocálicos forma diptongo. Este caso se da gracias a la poca perceptibilidad de una vocal semiabierta la que es reducida a una semiconsonante en diptongo creciente, como lo evidencian los siguientes casos:

1. 'Que hacer' $\rightarrow$ [kja 'ser]

2. 'Que había' $\rightarrow$ [kja ' $\beta i a]$

3. 'Me acuerdo' $\rightarrow$ [mja ' kwerdo]

4. 'De alambre' $\rightarrow$ [סja ' lambre]

5. 'Ocho años' $\rightarrow$ [ot $\int$ wano $]$ 
Rasgos de simplificación en el habla rural de dos localidades de Chile: descripción fonotáctica y discursiva / Dras@ Miriam Cid Uribe y Macarena Céspedes Morales

C. Con una significativa frecuencia se presencian casos de sinéresis, con lo que podemos afirmar que la diptongación es uno de los rasgos de mayor uso dentro de esta habla rural. Por ejemplo:
1. Emple.ado $\rightarrow$ [im ' pljado]
2. Espontáne.a $\rightarrow\left[\mathrm{e}^{\mathrm{h}}\right.$ pon 'tanja]

D. Por último damos cuenta de los ejemplos de sustitución encontrados en nuestro corpus de habla:
1. Legítima $\rightarrow$ [li ' xitima]
2. Madrugar $\rightarrow$ [mairu 'var]
3. Cabros $\rightarrow[$ ' kauro $\phi]$
4. Fui $\rightarrow\left[{ }^{\prime} \mathrm{x} w \mathrm{wi}\right]$

De los casos exhibidos, el más representativo de la realidad de habla rural, de Culiprán y Popeta, es la sustitución de consonante por vocal que se manifiesta cuando la variable oclusiva alveolar sonora / $d$ / en contacto con la vibrante simple alveolar $/ \mathrm{r} /$, es sustituida por la semivocal alta palatal [ $\mathrm{i}$ ]; en tanto que, la variable oclusiva bilabial/b/ en contacto con la vibrante simple alveolar $/ r$, es sustituida por la semivocal alta velar [u]. Ambos casos responden a la natural tendencia a producir habla realizando el mínimo esfuerzo articulatorio: la realización de una semivocal en diptongo decreciente requiere de menos complejidad articulatoria que una consonante oclusiva bilabial/ o alveolar en cambio de posición, dentro del tiempo de tensión, a la zona alveolar/ o cambio en el modo de oclusión a vibración simple.

\subsection{Rasgos discursivos en el habla de Culiprán y Popeta}

Uno de los rasgos discursivos observados en el habla de estas dos localidades rurales del país, es el alargamiento segmental que se clasificó en una primera instancia, dependiendo de si el caso fuese a) vocálico o b) consonántico y en una segunda instancia, se contempló la siguiente clasificación:

1. En monosílabos y vocalizaciones: a) $E h+, y+, n o+, u h+, d e+, l a+, q u e+$, se+, lo+(s), sít, me+, le+, yo+, mát(s), so+l+, fue+, mit, a+, sét, ta+n, $i+r$, oh+, se+, ya+, ve+r, quét, muyt, me+s, na+(da) b) Quien+, bien+, $m+e$, unt, $m+$, cont, sont, so $+l+$, elt

2. En la última sílaba de palabras bisílabas y polisílabas: a) Miedo+, iba+, unat, ahorat, eso+, porque+, perot, antiguamente+, ejemplo+, pilla+r, cuenta+, hace+r, bajonea+(da), campo+, poquito+, jugábamo+s, inventávamo+s, ello+s, mayo+r, allát, preocupa+(da), mejo+r, muriót, 
ahít, cambiót, despuéts, casa+(da), rega+r, muje+r, casét, cuesta+, tene+r, juventu+d, sali+r, disco+, tambié+n, jamá $+s$, computado $+r$, celula+r, igua+l, aquít, asít, papá+, raí +z, hostigót, gustó+, Culiprá+n, inventó+, quebra+(da), arriba+, valo+r b) Pensión+

3. Recurrentemente, en la sílaba tónica de palabras graves y esdrújulas: a) Respe+to, mayo+res, mete+rse, uni+dos, coci+na, insole+ntes, tecnologí+a, conta+ba, priva+do, ge+nte, desconoci+do, jugá+bamos, escondi+da, ponítan, difí+cil, ju+ego, hací+amos, galli+nas, decí+a, disfru+ta, gra+ndes, sa+le, ca+sa, pie+nso, gustaríta, pue+do, via+jo, dicie+ndo, profeso+ra, sue+ldo, tení+a, perdie+ron, nu+nca, vie+ja, recue+rdo, senci+llas, cole+gio, paya+ya, muñe+cas, to+dos, jorna+da, jue+ves, me+dios, le+jos, po+co, ha+rto, ju+ntos, domi+ngos, hací+a, hace+rlo, va+cas, caba+llos, relaja+do, disti+nto, co+mpro, chiquiti+ta, lava+ba, ca+ña, ce+rro

Se pudo observar que el alargamiento es un recurso discursivo recurrente del hablante rural, porque lo usa en diversas ocasiones dependiendo de la utilidad que le pueda dar al momento de producir habla espontánea.

1. En primer lugar, tenemos un alargamiento que se produce para llenar un vacío de contenido que es dependiente de la competencia lingüística del hablante, quien no siempre encuentra con facilidad la palabra precisa para elaborar sus enunciados y ocurre con naturalidad cuando se trata de alargar una sílaba abierta (Canellada:1987; 55), como lo demuestran los siguientes ejemplos: $a$-Si me voy pa(ra) otro lado yo no+ $\sim$ incluso he tenido hartas oportunidades pa(ra) cambiarme de pega, nunca me he querido ir. b- Ya ve el catoli(ci)smo+, todo eso, si te pones a analizar tú, es distinto al campo, claro. En estos enunciados el hablante deja inconclusa una secuencia textual, cerrando su idea con el alargamiento de la vocal abierta $O$; sin embargo, el sujeto logra continuar su acto de habla incorporando una secuencia explicativa que finaliza con éxito.

2. En segundo lugar y con menos frecuencia, observamos el alargamiento consonántico que se produce de acuerdo a nuestros resultados, solo en consonantes sonoras nasales y líquidas las que, además de llenar vacíos intensifican algunos elementos dentro de una narración, pongamos atención a los siguientes ejemplos: a- Porque ahora hay gente+, hombres viejos aquí en Culiprán que no son+ jubilados con la pensión+ legítima, claro, (...) b- Pero es católico; lleva el catoli(ci)smo en el+corazón, claro.(...) c- Ya despué+s cuando nosotros (es)tá+bamos gran+des (...) En los ejemplos a y b vemos cómo el hablante reposa su proceso articulatorio en una consonante, hasta que logra conti- 
Rasgos de simplificación en el habla rural de dos localidades de Chile: descripción fonotáctica y discursiva / Dras@ Miriam Cid Uribe y Macarena Céspedes Morales

nuar la producción de su enunciado; en cambio, en el ejemplo c se puede observar que el alargamiento de la consonantes $n$ intensifica el significado de este grupo fónico.

3. Por último, el alargamiento encontrado en la sílaba tónica de la palabra se presenta principalmente en enumeraciones dentro de los enunciados, por ejemplo: a- Al pilla+r, a la escondi+da, eh+ (...) b- En cambio, creo yo que los de la ciudad, se llevan en los jue+gos, eh+, en las di+scos de jovencito como que+ $~$ es diferente. (...) Estas marcas de alargamiento reflejan el estilo discursivo del habla rural, que es menos fluido que el urbano (siendo igual o tanto más rápida la producción de los segmentos) y posee una velocidad secuencial no acelerada como ocurre, generalmente, en las hablas urbanas ${ }^{4}$.

\section{Conclusiones}

Nos atrevemos aquí a postular que aunque el habla rural de Chile comparte los mismos fonemas del habla estándar, no presenta convergencia en todas las realizaciones alofónicas de esta última variedad y presenta, además, una distribución fonológica distinta en su combinación y utilización de los segmentos de la lengua, lo que la caracteriza y distingue como una variedad lingüistica (dialectal) independiente.

Al analizar este primer postulado a la luz de los datos empíricos obtenidos del estudio de un corpus de habla real recolectado en las localidades que hemos mencionado, pudimos observar la ocurrencia y recurrencia de un amplio repertorio de rasgos de simplificación que se corresponden con los elementos fonotácticos del habla. Tales rasgos de simplificación parecieran encontrarse con mayor recurrencia en los tiempos de intensión y distensión del proceso de articulación del habla: en la variedad bajo estudio, el tiempo de tensión es menos extenso que en el habla estándar, característica que afecta el tempo del primero. El habla rural producida en las localidades de Culiprán y Popeta aparece, entonces, caracterizada por la ocurrencia en un alto grado de elisiones, asimilaciones, sinalefas y sustituciones, los que reflejan particularidades motivadas por la necesidad de acceder a un nivel de competencia lingüística esperable en cualquier usuario de la variedad estándar de Chile.

Sin embargo, la recurrencia de los rasgos de simplificación en el habla rural no parecieran diferir de la recurrencia de los fenómenos

4 En este caso nos referimos a la velocidad que tardan en construir los textos orales, independiente de la velocidad de producción de los fonemas una vez que la secuencia textual ha sido superada. 
de simplificación en el habla estándar; por lo tanto, las características primordiales del comportamiento de estos rasgos en el habla rural tienen que ser encontrados no en los rasgos fonotácticos persé, sino en la relación entre tales rasgos, los rasgos segmentales -fonemas o alófonos-, los rasgos suprasegmentales o prosódicos -acento, acentuación, ritmo, entonación-y los rasgos paralingüísticos que acompañan el habla -cantidad y calidad de voz- los que acompañados de una altura tonal característica y de un tempo también característico resultan en un perfil de habla propio de la variedad.

Además, y como una conclusión que proyecta otras investigaciones para el futuro, podemos decir que, al analizar el flujo espontáneo del habla e intentar reproducirla en un medio visual más que temporal a través de un sistema de transcripciones, queda en evidencia que tal sistema -cualquiera sea y sin importar cuán estrecha resulte ser la transcripción- resulta incapaz de capturar muchos de los comportamientos que se producen en el devenir discursivo de la oralidad. Nos atrevemos, entonces, a concluir predictivamente que para proponer una descripción acabada de cualquier variedad de habla, en general, y del habla rural de Chile, en particular, y postular una caracterización identificatoria de esta última, será necesario observar y describir la interacción de todos los elementos prosódicos, paralingüísticos y segmentales según ellos se imbrican para lograr un mensaje coherente, cohesivo y caracterizador.

\section{Bibliografía}

Álvarez, Alexandra, (1996). Acerca de los Estudios sociolingüísticos en Venezuela. Universidad de los Andes. Álvarez, Alexandra y Thania Villamizar, (1999). Las variantes fonéticas rurales del habla de la Cordillera de Mérida: ¿Cambio lingüístico o patrón de sexo? Lengua y habla. Universidad de los Andes.

Cid Uribe, Miriam, (2003). Descripción fonofonológica de los patrones prosódicos recurrentes en los actos de habla pública de Chile: proyecto de investigación. P. U. C. Revista Onomázein.

Silva-Corvalán, Carmen, (2001).

Sociolingüística y pragmática del español / Carmen Silva-Corvalán; con ejercicios de reflexión de Andrés Enrique-Arias. Washington: Georgetown University Press.

López Morales, Humberto, (2004).

Sociolingüística. Madrid: Gredos. 
Rasgos de simplificación en el habla rural de dos localidades de Chile: descripción fonotáctica y discursiva / Dras@ Miriam Cid Uribe y Macarena Céspedes Morales

López Morales, Humberto, (1994).

Métodos de investigación lingüística. Salamanca:

Colegio de España.

Llisterri Boix, Joaquín, (1991).

Introducción a la fonética: el método experimental. Barcelona: Anthropos.

Moreno Fernández, Francisco, (2005).

Principios de sociolingüística y sociología del lenguaje. Barcelona: Ariel.

Obediente, Enrique, (1992). El habla rural de la Cordillera de Mérida: Léxico y Fonetismo. Boletín Antropológico, Mérida: ULA.

Obediente, Enrique. (1996). Datos sobre la R asibilada en Venezuela. Lengua y Habla. Mérida: ULA.

Quilis, Antonio, (1999). Tratado de fonología y fonética españolas. Bliblioteca románica hispánica, Ed. Madrid: Gredos.

Roldán Yánez, Eduardo, (2000).

Rasgos de calidad de la voz en el habla rural chilena. Artículo del Boletín de Investigación Educacional, vol. 15. 